\title{
Determinants of Gender- based Violence Against Women in Spain: An Asymmetric Bayesian Model
}

Journal of Interpersonal Violence

$1-20$

(C) The Author(s) 2020

Article reuse guidelines: sagepub.com/journals-permissions DOI: I0.II77/0886260520967749 journals.sagepub.com/home/jiv

@SAGE

\section{José María Pérez-Sánchez, ' (iD Nancy Dávila- Cárdenes, ' and Emilio Gómez-Déniz'}

\begin{abstract}
The number of fatalities in Spain due to gender-based violence has increased in recent years, with a new rise in 2019 , reaching the highest figure since 2015 , a year that registered a peak with 60 victims. This article analyzes a database obtained from a survey on gender violence conducted by the Spanish Centre for Sociological Research. The survey, prepared by the Government Delegation for Gender Violence, consisted of interviews with women aged over 15 years living in 858 municipalities distributed over 50 provinces in Spain. The data reveal that most of the women interviewed have not suffered any type of physical, sexual, or psychological abuse. Hence, the application of standard logistic methodologies which suppose symmetric responses, can lead to a poor specification of the model, a misinterpretation of marginal effects and unidentified predictors. It seems more appropriate to consider an asymmetric link function to explain the probability of abuse (physical, sexual, or psychological). The Bayesian methodology allows the incorporation of such an asymmetric function improving the specification of the model. In this article, we compare both methodologies and prove that Bayesian asymmetric performs better results by considering several diagnostic
\end{abstract}

'Gran Canaria, Las Palmas, Spain.

\section{Corresponding Author:}

José María Pérez-Sánchez, University of Las Palmas de Gran Canaria, 3500I Las Palmas de Gran Canaria, Las Palmas, Spain.

E-mail: josemaria.perez@ulpgc.es 
criteria. Furthermore, this methodology detects some significative factors that are not revealed by the classical method, e.g., the partner's nationality for sexual abuse or the women's total number of intimate partners for psychological abuse. Bayesian asymmetric estimations reveal no significance concerning to the lowest partner's level of education for physical abuse but if the intimate partner is currently studying this reduces the probability of sexual abuse. The woman's level of education is not relevant to the physical, sexual, or psychological abuses suffered. Therefore, the findings may help identify economic and sociological factors not previously considered in this area and highlight policies that may be adopted or revised to help overcome this social problem.

\section{Keywords}

gender-based violence against women, asymmetric logit regression, Bayesian estimation, intimate partner

\section{Introduction}

Gender-based violence (GBV) and violence against women (VAW) is an ongoing concern throughout the world (Allen et al., 2018; Goodey, 2017; Johnson, 2006), and much research is devoted to understanding the factors that may influence the likelihood that a women experiences GBV (Capaldi et al., 2008). In this article, we analyze data from the VAW survey conducted by the Spanish Centre for Sociological Research (CIS) in late 2014. These data reveal that most of the women interviewed do not suffer physical, sexual, or psychological abuse. The use of a symmetric link function as developed in both classical and Bayesian logit specification is recommended for binary response data in which the frequency of one response is similar to the frequency of the other response. If this is not the case, then an analysis using an asymmetric link function is preferable (Chen et al., 1999). Apart from Chen et al. (1999)'s work, the statistical literature provides other proposals that have followed the line developed in that work. Some of these are Kim et al. (2008), Wang and Dey (2010), Jiang et al. (2013) and Lemonte and Bazán (2018).

In the Bayesian approach, the regression coefficients are considered to be random variables assuming noninformative and centered normal prior distributions for the $\beta$ coefficients in order to facilitate comparisons with classical estimations (see, for instance, Zellner [1996, Chapter 3] and Koop [2003, Chapter 2]). The advantage of Bayesian methods is that it incorporates not only the data at hand but also prior accumulated knowledge to generate 
parameter estimates that tend to be different and more accurate than those derived under classical methods. An interesting and pedagogical interpretation of this can be seen in Koop (2003, Chapter 2).

Classical statistics, based mainly on the estimation from the collected data, provide tools to carry out the objective that is intended to be addressed in this work. However, given the nature of the data (the asymmetry in our case), the use of the classical methodology may not be able to define elements that are affordable with the use of the Bayesian methodology, which can use, apart from the data, prior information provided by a well-trained practitioner. Bayesian inference for logistic analyses follows the usual pattern in Bayesian analysis consisting of the likelihood function of the data, the prior distribution over the unknown parameters and the Bayes' theorem to compute the posterior distribution of the parameters. There exists in the literature a lot of procedures about choosing the prior distributions based on noninformative, default, and reference prior distributions. See, for example, Jeffreys (1961), Kass and Wasserman (1996), Spiegelhalter and Smith (2002), Bernardo (2004) and Hartigan (2014), among others. For a comprehensive knowledge about this topic, the reader can consult the paper of Gelman et al. (2008). The most common priors for logistic regression parameters are $\beta_{j} \sim N\left(\mu_{j}, \sigma_{j}^{2}\right), j=1, \ldots, k$; where $\mu_{j}$ are usually supposed to be zero and $\sigma_{j}$ are commonly chosen to be large enough to be considered noninformative. In this article, we consider these priors to facilitate comparisons between classical and Bayesian estimations.

In this study, the selection of socioeconomic variables was based on previous research. Farmer and Tiefenthaler (1997), Yodanis (2004), Famoye and Singh (2006), Aizer (2010), Capaldi et al. (2008), Bhattacharyya et al. (2011), and Alonso-Borrego and Carrasco (2017) analyze factors regarding the domestic violence as employment and property status, employment income, educational and occupational status, the impact of sexual male-female wage gap, among many others.

The rest of this article is organized as follows. The database used in the analysis is described in Section 2. The theoretical aspects of the logistic regression models used are presented in Section 3. In Section 4, we discuss the results obtained and study some diagnostic measures to determine whether the symmetric or the asymmetric model is preferable for our purposes. Finally, we present the main conclusions drawn.

\section{Data Collection}

The data used in this study were obtained from a nationwide CIS survey conducted at the end of 2014. The survey, prepared by the Government Delegation 
for Gender Violence, consisted of interviews with women aged over 15 years living in 858 municipalities distributed over 50 provinces in Spain. The initial aim was to interview 10,258 women, but full responses were only obtained from 5,818 women, although these accounted for 10,171 observations. The sampling allocation method was nonproportional. The sampling procedure was multistage, stratified by clusters via a questionnaire presented in a personal interview in the participant's home. This article considers three models with physical, sexual, and psychological abuses as dependent variables representing abuse by the current or most recent intimate partner in the last twelve months. These three dependent variables are based on a set of different questions from the original survey for the three type of abuses. If the response was "never," the variable was codified by 0 ; and 1 otherwise ("once," "sometimes," and "many times").

The explanatory variables for the three models were selected following two steps. In the first one, 41 variables extracted from the survey were chosen attending socioeconomic reasons or in terms of policy implications. The second step consisted in applying Bayesian model averaging (see Hoeting et al., 1990, and references therein for a comprehensive study of this technique) from the previous 41 covariates and over $10 \cdot 10^{11}$ competing models, after confirming the absence of collinearity according to the variance inflate factor and ensuring the independence of the explanatory variables. Selected variables for analysis were classified into four categories: abuse, economic, sociodemographic, and academic indicators. The abuse indicators included, on the one hand, physical and sexual abuse from anyone who had never been an intimate partner of the woman and, and on the other hand, the fact that the current (or most recent) intimate partner is (or was) jealous. The economic indicators included the GDP per capita in the region (in $€$ ), the monthly household income after tax (in $€$ ), the employment situation of the current (or most recent) intimate partner, the household member who contributed most to joint income, i.e. the woman or the intimate partner, and the kind of income earned, i.e., casual, permanent, or unregulated. The sociodemographic indicators were the size of the municipality (on a scale ranging from less than two thousand to over one million inhabitants), whether the woman had children, the number of intimate partners in her lifetime, the current existence or otherwise of an intimate partner, the partner's gender and nationality, and the age of the woman and of her partner. Finally, the academic indicators included the partner's educational background, whether the partner was currently studying and the woman's educational background.

The survey data are available on the CIS web page. The sample error has a $95.5 \%$ level of confidence and the real error is $0.99 \%$ for the whole sample 
Table I. Descriptive Summary of Quantitative Variables.

\begin{tabular}{lcccccc}
\hline Variable & Min & Max & Mean & $P_{25}$ & $P_{50}$ & $P_{75}$ \\
\hline GDP per capita & 16,166 & 31,812 & 22821.07 & 18,354 & 20,586 & 27,663 \\
Household income & 0 & 7,500 & 1582.47 & 750 & 1,500 & 2,100 \\
Size of municipality & 1,000 & $2,386,500$ & 340149.2 & 30,000 & 75,000 & 250,000 \\
Number of partners & $\mathrm{I}$ & 20 & 1.652 & $\mathrm{I}$ & $\mathrm{I}$ & 2 \\
Age of the woman & 16 & 94 & 48.64 & 36 & 47 & 61 \\
Age of the partner & 15 & 96 & 49.57 & 37 & 48 & 62 \\
\hline
\end{tabular}

Table 2. Descriptive Summary of Categorical Variables (Absence or Presence).

\begin{tabular}{|c|c|c|}
\hline Variable & Yes (I) & No $(0)$ \\
\hline Physical abuse & $270(4.64 \%)$ & $5,548(95.36 \%)$ \\
\hline Sexual abuse & $235(4.03 \%)$ & $5,583(95.97 \%)$ \\
\hline Psychological abuse & 867 (14.90\%) & $4,951(85.10 \%)$ \\
\hline Physical abuse not partner & $690(11.85 \%)$ & $5,218(88.15 \%)$ \\
\hline Sexual abuse not partner & $432(7.42 \%)$ & $5,386(92.58 \%)$ \\
\hline Jealous & $\mathrm{I}, 044(\mathrm{I} 7.94 \%)$ & $4,774(82.06 \%)$ \\
\hline Partner employment & $3,509(60.31 \%)$ & 2,309 (39.69\%) \\
\hline Women contributed most to joint income & $2,373(40.79 \%)$ & $3,445(59.21 \%)$ \\
\hline Partner contributed most to joint income & $4,146(7 \mid .26 \%)$ & $\mathrm{I}, 672(28.74 \%)$ \\
\hline Casual salary & $\mathrm{I}, 625(27.93 \%)$ & $4,193(72.07 \%)$ \\
\hline Permanent salary & $3,318(57.02 \%)$ & $2,500(42.98 \%)$ \\
\hline Other salary & $102(1.75 \%)$ & $5,716(98.25 \%)$ \\
\hline Children & $4,745(81.55 \%)$ & $\mathrm{I}, 073(\mathrm{I} 8.45 \%)$ \\
\hline Current partner & $4,626(79.51 \%)$ & $\mathrm{I}, \mathrm{I} 92(20.49 \%)$ \\
\hline Partner's gender (I if woman) & $5,787(99.47 \%)$ & 31 (0.53\%) \\
\hline Partner's nationality (I if Spanish) & $5, \mid 70(88.86 \%)$ & 648 (II.14\%) \\
\hline Partner's level of studies under 5 years & $392(6.73 \%)$ & $5,426(93.27 \%)$ \\
\hline $\begin{array}{l}\text { Partner's level of studies at primary } \\
\text { education or vocational training }\end{array}$ & $1,340(23.04 \%)$ & $4,478(76.96 \%)$ \\
\hline Partner's level of studies at high studies & $1,016(17.46 \%)$ & $4,802(82.54 \%)$ \\
\hline Partner currently studying & $133(2.28 \%)$ & $5,685(97.72 \%)$ \\
\hline Woman's level of studies at high studies & $\mathrm{I}, \mathrm{I} 27$ (19.37\%) & $4,691(80.63 \%)$ \\
\hline
\end{tabular}

in simple random sampling. A descriptive study of all these variables in the sample is shown in Tables 1 and 2. 


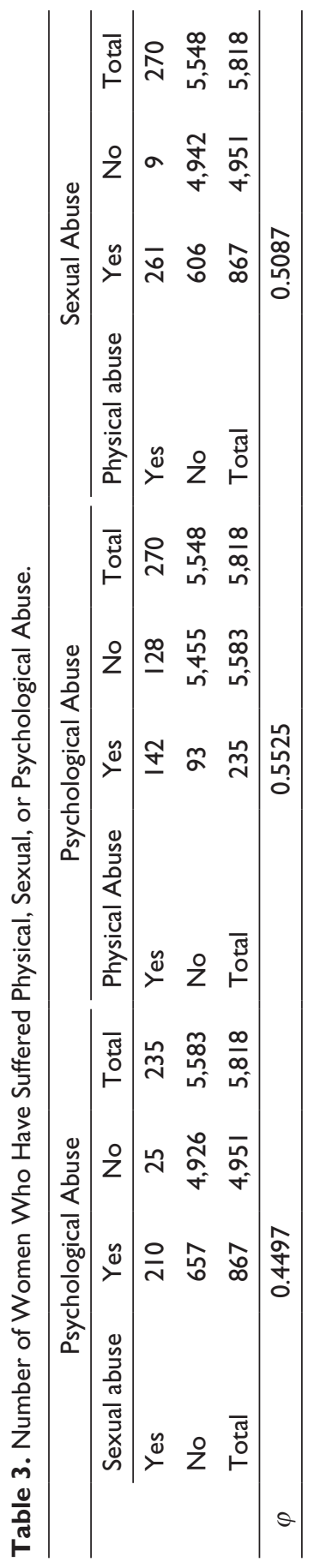


Table 3 reports the $\phi$ coefficient, also known as the mean square contingency, which is a measure of association for two binary variables and is interpreted in a similar way to the Pearson correlation coefficient. The results obtained present strong positive correlations among the three dependent variables, which are analyzed as follows.

\section{Statistical Methods}

When a research context produces binary outcomes, it is usually analyzed by logit and/or probit models. A binary response model is a regression model in which the dependent variable $y$ is a random binary variable that takes only the values zero or one.

In this study, we use the logit model to estimate the probability of abuse (physical, sexual or psychological) given a set of characteristics of the event; that is, given the predictor $X$, we estimate the conditional probability $\operatorname{Pr}(1 \mid X=x)$. Specifically, we use a symmetric logit model and an asymmetric Bayesian model and then, we compare the results of the two estimations.

\section{Symmetric Assumption}

Specifically, the classical (and symmetric) logit is defined as follows. For observation $t$ in a sample of size $n$, let $y_{t}, t=1,2, \ldots, n$, a binary variable taking the value of 1 with probability

$$
p_{t}=\frac{1}{1+\exp \left(-x_{t}^{\prime} \beta\right)}=\frac{\exp \left(x_{t}^{\prime} \beta\right)}{1+\exp \left(x_{t}^{\prime} \beta\right)},
$$

and 0 with probability $1-\mathrm{pt}$, where $\beta=\left(\beta_{1}, \beta_{2}, \ldots, \beta_{k}\right)^{\prime}$ is a $\mathrm{kx} 1$ vector of regression coefficients which represents the effect of each variable in the model and it should be estimated. Finally, $x_{t}=\left(x_{t 1}, x_{t 2}, \ldots, x_{t k}\right)^{\prime}$ is a vector (explanatory variables) of known constants, including an intercept, the vector of covariates. The regression is therefore modelled by assuming that $p_{t}=F\left(x_{t}^{\prime} \beta\right)$, where $\mathrm{F}$ is the inverse of the standard logistic cumulative function, the link function, a function which relates the response variable to the predictors in the model. Recall that the probability density function of the standard logistic distribution is symmetric about 0 . In summary, the classical logit specification adopts the following form:

$$
L_{t}(\beta)=\log \left(\frac{p_{t}}{1-p_{t}}\right)=x_{t}^{\prime} \beta, t=1,2, \ldots, n .
$$




\section{Asymmetric Assumption}

In Table 2, it can be seen that the answer 0 (and therefore they stated that they do not suffer any type of abuse) is much more frequent than the 1 (they answered yes to some type of abuse). Hence, it seems more appropriate to consider an asymmetric link function to explain the conditional probability response variable $\operatorname{Pr}(1 \mid X=x)$. In this case, the application of the previous classic model can lead to a poor specification of the model and a misinterpretation of marginal effects and unidentified predictors.

From the asymmetric point of view, an approach based on data augmentation, as considered by Albert and Chib (1995), can be used. In this way, it is easily shown (see Chen et al., 1999) that a skewed logit link is equivalent to considering that

$$
y_{t}= \begin{cases}1, & w_{t} \geq 0 \\ 0, & w_{t}<0\end{cases}
$$

where $w_{t}=x_{t}{ }^{\prime} \beta+\delta z_{t}+\varepsilon_{t}, z_{t} \sim G, \varepsilon_{t} \sim F$. In this model, $\delta \in(-\infty, \infty)$ is the skewness parameter and so the skewness of the regression model is measured by $\delta z_{t}$. If $\delta>0$, the probability of $p_{t}=1$, the probability that the $t$ th woman abuse, increases. On the other hand, if $\delta<0$, the probability of not abusing increases.

The new Bayesian asymmetric logit model can be written as follows:

$$
\begin{aligned}
& L_{t}(\beta, \delta)=\log \left(\frac{p_{t}}{1-p_{t}}\right)=x_{t}^{\prime} \beta+\delta z_{t}, \\
& (\beta, \delta) \sim \pi(\beta, \delta),
\end{aligned}
$$

where $\pi(\beta, \delta)$ is a bivariate prior distribution for $(\beta, \delta)$. From model in (4)-(5), it can be easily seen that the symmetric logit model given in (2) can be considered as a particular case of the previous model when $\delta=0$. We assume that $z_{t}$ and $\varepsilon_{t}$ are independent and that $F$ is the standard logistic cumulative distribution function. Moreover, $G$ is the cumulative distribution function of the half-standard normal distribution given by $g(z)=\frac{2}{\sqrt{2 \pi}} \exp \left(-\frac{z^{2}}{2}\right), z \geq 0$.

\section{Results}

The models for physical, sexual, and psychological abuse were estimated. For this purpose, we have considered the model in (2) for the classical methodology and the model in (4)-(5) for the Bayesian methodology. The results 
obtained by the classical and asymmetric Bayesian estimations are shown in Tables 4-6, respectively. These tables show the estimated coefficients, $\hat{\beta}$, robust standard deviations, $p$ values and the average elasticities, $\bar{\varepsilon}$, for the classical estimations, and the estimated coefficients, standard deviations, MC errors and the average elasticities for the asymmetric Bayesian estimations. To normalize the variables' units, elasticities were calculated in the following form:

$$
\varepsilon_{t x_{j}}=\widehat{\beta}_{j} \bar{x}_{j}\left(1-\hat{p}_{t}\right),
$$

where $\bar{x}_{j}$ is the mean of $x_{j}(j=1, \ldots, k)$ and $\hat{p}_{t}$ is the estimated probability for each observation of the sample. Finally, the tables show the mean of these elasticities for each variable, i.e., $\bar{\varepsilon}$. To perform the Bayesian estimation procedure, the noninformative prior distributions must be determined. For this purpose, we assume $\sigma_{j}^{2}=10^{8}, \forall j=1, \ldots, k$, and $\sigma_{\delta}^{2}=10^{8}$. These high values cover a broad spectrum of situations since the prior distributions are flat and can be considered vague priors. Haining and Li (2020, Chapter 5, Section 6) illustrates various situations similar to the one shown in this work. See also Spiegelhalter et al. (2002) and Spiegelhalter and Smith (2002) for further information. The posterior distributions for the Bayesian models were simulated using WinBUGS in the three samples. A total of 500,000 iterations were carried out (after a burn-in period of 100,000 simulations) for each sample. Three different chains were performed, and the convergence was evaluated for all parameters using tests provided within the WinBUGS Convergence Diagnostics and Output Analysis software.

\section{Physical Abuse}

Table 4 shows the estimation results for physical abuse. According to the classical and asymmetric Bayesian estimations, the intercept, the existence of sexual or psychological abuse and the nationality of the intimate partner are all statistically significant in explaining the probability of physical abuse, at $1 \%$ significance. Hence, if there is sexual and/or psychological abuse, the probability of physical abuse increases, but if the intimate partner is of Spanish nationality, this probability decreases. If the woman has children and if the current intimate partner (or the most recent one) is jealous, these factors are positively associated and statistically significant at 5\% significance. However, if the woman has an intimate partner at the time of the survey, the probability of physical abuse decreases. The fact that the breadwinner is a casual worker increases the probability of the woman suffering physical 


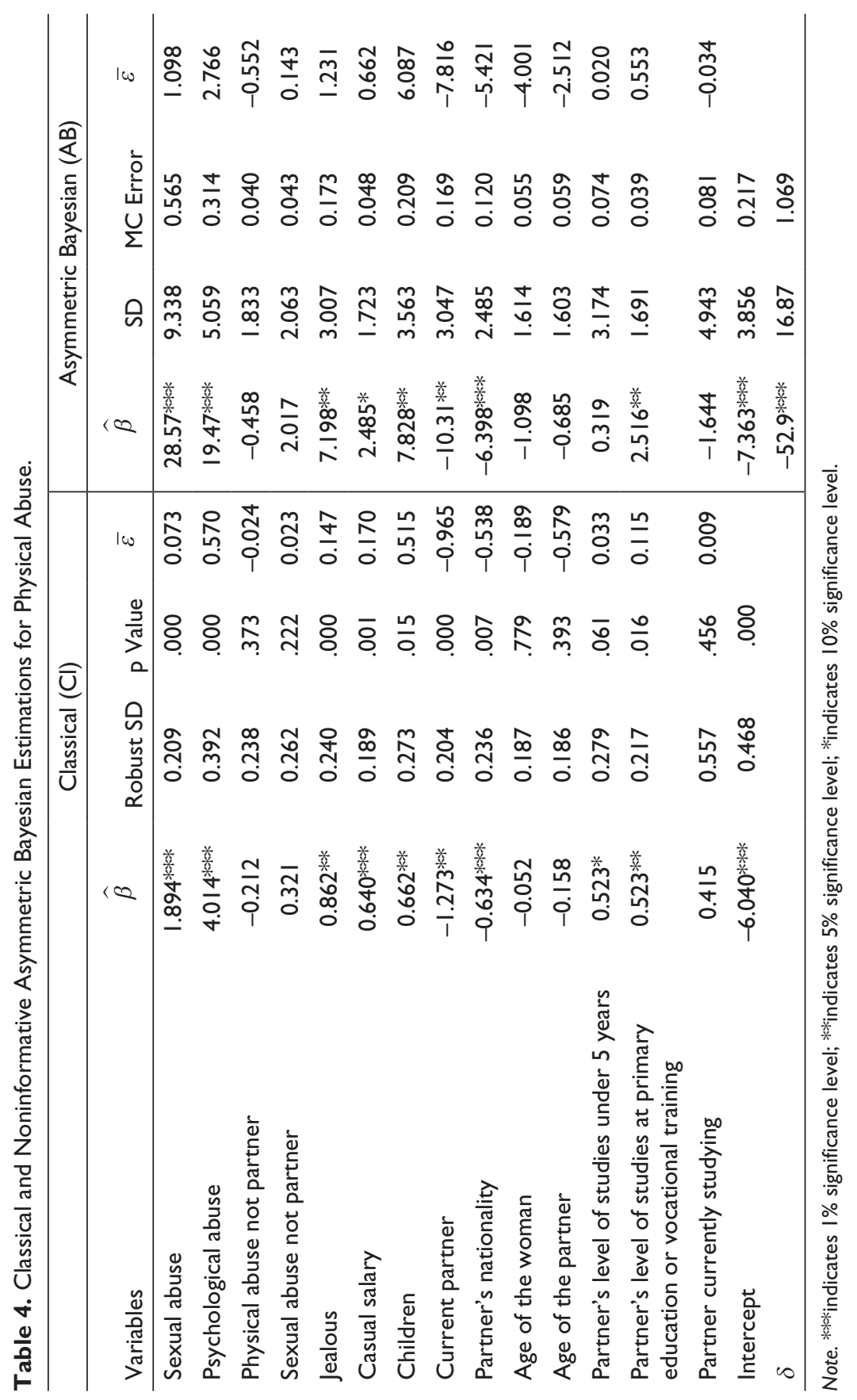




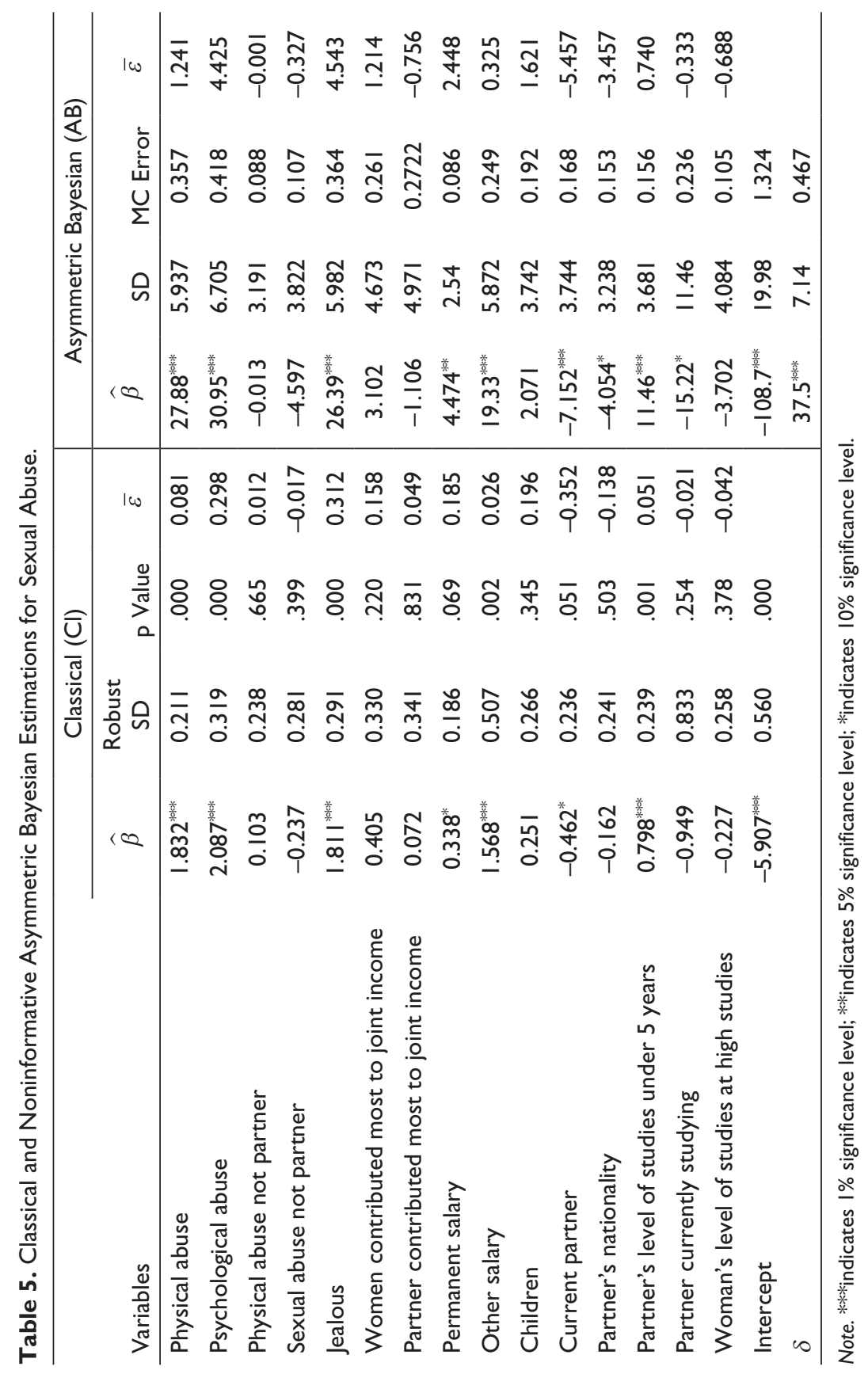


abuse, at $1 \%$ and $10 \%$ significance, from the classical and asymmetric Bayesian standpoints, respectively. Finally, the classical estimation detects that the intimate partner's educational background is a significant factor. More precisely, if the partner has less than five years' formal education, or has only primary or vocational training, the probability of the woman being physically abused is greater than that of women whose partners have higher education qualifications, at $10 \%$ and $5 \%$ significance, respectively. In this sense, the Bayesian asymmetric model detects only the primary or vocational training as a significant factor. According to the information provided by the elasticities, the fact of having currently a partner is the most important factor explaining the probability of physical abuse for both the classical and the Bayesian models. The variable $\delta$, which measures the skewness of the data, is statistically significant and negative at $1 \%$ significance, i.e, decreasing the probability of physical abuse and correcting the evident asymmetry in the data. The estimated intercept in the classical model may contain part of the asymmetry effect that is apparent in the asymmetric model.

\section{Sexual Abuse}

The results obtained for sexual abuse are shown in Table 5. According to the classical and asymmetric Bayesian estimations, the intercept, the existence of physical or psychological abuse, that of jealousy, unregulated wages and the intimate partner's low level of education are all associated positively and significantly with the probability of sexual abuse, at $1 \%$ significance. A stable household income is also positively and significantly associated with sexual abuse, at $10 \%$ and $5 \%$ significance, from the classical and Bayesian standpoints, respectively. However, if the woman had an intimate partner at the time of the survey, the probability of sexual abuse was lower. The Bayesian asymmetric model revealed two factors that had remained undetected by the classical model, namely if the intimate partner was currently studying, and the nationality of the partner. Both variables were associated with a lower probability of sexual abuse. As in the physical abuse, in percent changes, the most important determinant for the sexual abuse is having an intimate partner at the time of the survey. The variable $\delta$ is again significant, at $1 \%$, which justifies the use of the asymmetric model.

\section{Psychological Abuse}

Table 6 shows the estimation results for psychological abuse. According to the classical and asymmetric Bayesian estimations, the intercept, the existence of physical or sexual abuse (by an intimate partner or anyone else), 


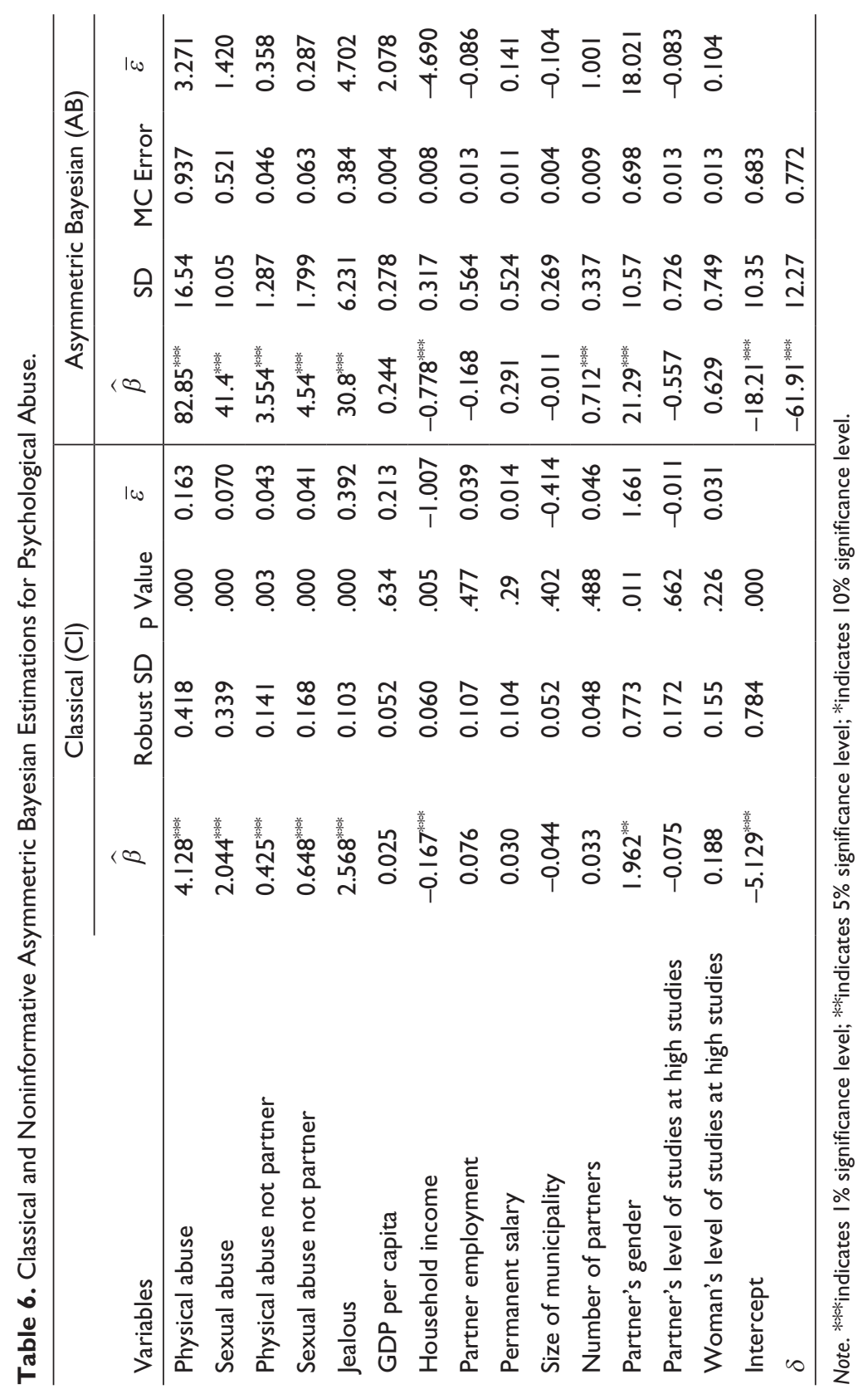


income and jealousy are all statistically significant in explaining the probability of psychological abuse at 1\% significance. Except for the intercept and the family income, all these variables are associated with an increased probability of abuse. The probability of psychological abuse decreases as the household income increases. Furthermore, if the current (or most recent) intimate partner is (was) a man, this increases the probability of psychological abuse from the classical and Bayesian standpoints, at 5\% and $1 \%$ significance, respectively. Finally, Bayesian estimation detects a new risk factor in psychological abuse that is not revealed in the classical approach, namely that the number of intimate partners during the women's lifetime is positively associated with the probability of psychological abuse, at $1 \%$ significance. A percent change in the average proportion of heterosexual partners is associated with the most important percent change in the probability of psychological abuse. Again, the variable $\delta$ is significant, at $1 \%$, which justifies the use of the asymmetric model.

\section{Comparative Diagnostics}

The goal of a regression model, such as the logistic model considered here, is to correctly fit and predict the category of outcome for individual cases using the best model and including all the predictor variables considered to be useful in explaining the response variable. To assess the goodness of fit obtained by the classical and the Bayesian logit models, three different measures were applied:(a) the percentage of correct fits, calculated by considering the estimated probabilities; (b) the $c$-statistic which measures the goodness of fit in the logistic model curve; and (c) two statistical fit measures, the Akaike information criterion (AIC), defined as $\mathrm{AIC}=2(k-\log (\ell(y \mid x, \widehat{\beta})))$, see Akaike (1974) for further details, and the deviance information criterion (DIC), given by $D(\beta)=-2 \log (l(y \mid x, \widehat{\beta}))$ and described in Spiegelhalter et al. (2002). Here, $\widehat{\beta}$ are the estimated parameters that are usually obtained by maximum likelihood estimation. Both statistics measure the relative quality of statistical models for a given set of data. The idea is that models with smaller AIC and DIC are preferable to those with larger ones. Other measures such as the Expected Akaike Information Criterion (EAIC), the HannanQuinn Information Criterion (HQIC), the Expected Bayesian Information (Schwarz) Criterion (EBIC) proposed in Brooks (2009) and the sum-ofsquares of latent residuals (SSLR) can also be considered. A detailed description of some of these criteria can be found in Spiegelhalter et al. (2014).

Results provided in Table 7 reveals that the asymmetric link model is better in the three settings considered when assuming AIC, DIC, percentage of 
Table 7. Diagnostic Results.

\begin{tabular}{lcccccc}
\hline & \multicolumn{2}{c}{ Physical Abuse } & \multicolumn{2}{c}{ Sexual Abuse } & \multicolumn{2}{c}{ Psychological Abuse } \\
\cline { 2 - 7 } & $\mathrm{Cl}$ & $\mathrm{AB}$ & $\mathrm{Cl}$ & $\mathrm{AB}$ & $\mathrm{Cl}$ & $\mathrm{AB}$ \\
\hline AIC & 922.895 & 151.200 & 1019.843 & 101.400 & 2950.247 & 467.300 \\
DIC & 892.895 & 207.684 & 987.843 & 136.672 & 2920.247 & 768.087 \\
\% correct fit & 96.67 & 99.91 & 96.70 & 99.17 & 90.45 & 100 \\
c-statistic & 0.962 & 0.985 & 0.993 & 0.997 & 0.866 & 0.881 \\
\hline
\end{tabular}

Note. $\mathrm{AIC}=$ Akaike information criterion; $\mathrm{DIC}=$ deviance information criterion .

correct fit and $c$-statistic as criteria of comparison. For our database and physical abuse, we obtained an AIC of 922.895 , a DIC of 892.895 and a correct fit rate of $96.67 \%$ for the classical logit model. With asymmetric Bayesian estimation, the AIC and DIC were 151.2 and 207.684, respectively, i.e., the information criteria values obtained for the asymmetric model were notably lower than the ones obtained for the classical model. The major reduction in these measures indicates a significant increase in the level of fit. Furthermore, the asymmetric model obtained better classification results, with $99.91 \%$ correct fit (only 5 fails from a sample of 5,818 observations). Thus, the leverage of this model is much better than the classical one. The threshold probability used to fit the physical abuse was the sample frequency, namely 0.0464 . In the case of sexual abuse, the AIC is 1019.843 , the DIC is 987.843 and the correct fit rate is $96.70 \%$ for the classical logit model. With asymmetric Bayesian estimation, the AIC and DIC were 101.4 and 134.672, respectively. The asymmetric model obtains better classification results, with $99.17 \%$ correct fit failing in 48 observations. The threshold probability used to fit the sexual abuse was the sample frequency, namely 0.0403 . Finally, for the psychological abuse the AIC is 2950.247 , the DIC is 2920.247 and the correct fit rate is $90.45 \%$ for the classical logit model. With asymmetric Bayesian estimation, the AIC and DIC were 467.3 and 768.087, respectively. The asymmetric model obtains better classification results, with $100 \%$ of correct fit. The threshold probability used to fit the psychological abuse was the sample frequency, namely 0.1490 . In all the cases, the $c$-statistics choose the asymmetric Bayesian estimations as the best ones to fit the data.

\section{Discussion and Conclusions}

This article studies gender-based violence which is a topical issue in both developed and developing countries using data supplied by the Centre for Sociological Research (CIS) and the Government Delegation for Gender Violence in Spain. We identify the factors involved in physical, sexual, and 
psychological VAW in Spain, a problem that affects women of all ages and conditions. Taking into account that most of the women interviewed do not suffer any type of abuse, the classical logistic and Bayesian regression models, which assumes similar proportions in the response variable, do not seem to be appropriate for determining the factors or determinants underlying this phenomenon. Accordingly, an asymmetric logistic regression model was applied by detecting some factors that are not revealed by the classical methods, including sociological factors, and therefore contributes to the design of better-targeted policies with which to address this problem.

This type of study is adequate not only in the scenario we are considering but in all those in which there is a marked difference between the two types of responses that constitute the endogenous variable. So, a prudent researcher should, in our view, analyze the data prior to the study and then use the model that best suits them. Obviously, a similar proportion in the two responses of the endogenous variable would not require a study beyond the classical logit, but the use of a model of this nature when there is a marked asymmetry in the responses could be ignoring elements that considerably change the conclusions of the study.

The asymmetric model includes the classic logit model as a particular case and the results obtained by both are included, which can be useful for comparisons. In particular, the results obtained here indicate that the asymmetric link produces, apart from other comparative diagnostics, a better fit than the symmetric model and, therefore, seems more appropriate for analyzing this sort of data. In this sense, asymmetric Bayesian estimation isolates the asymmetric nature of the data and not only reveals new determinants but also the elasticities highlight the marginal effects in a more reliable way. Regarding physical abuse, asymmetric Bayesian estimations find the same significant factors as classical model, except for the partner's level of studies under 5 years which is not important for the asymmetric Bayesian model. In relation to sexual abuse, the asymmetric Bayesian model detects two new significant factors, i.e., the partner's nationality and the fact that this person is (or not) currently studying. Finally, regarding psychological abuse, the results are again similar, except for the number of partners which is a significant determinant for the asymmetric Bayesian estimations.

These findings undoubtedly help to identify economic and sociological factors that have probably not been considered in the past when addressing this social problem. Recently, in their ministerial offices, most developed countries have incorporated specialist teams to tackle the issue of gender violence. There is no doubt that these findings should be considered by political authorities in the moment of implementing measures to solve this problem, adapting, or revising the existing policies so far. 
These results could be useful not only in the area of violence towards women in intimate relationships (which has been addressed here) and in other areas where abuse responds to a network of patriarchal violence. Finally, regardless of the type of maltreatment suffered, it seems that the cultural element plays, as it seemed predictable, a fundamental role for both, the person who commits the abuse, and the woman who suffers it. Then, the gender policies carried out should be accompanied by actions from the educational field that promote gender equality.

The nature of this study analyzes only women residents in Spain. However, the women who responded in the sample had different socioeconomic statuses, races, ethnicities, languages, nationalities, gender identities, sexual orientations, religions, geographies, abilities, and ages. In general, these characteristics are not significant when studying any type of abuse in relation to the women interviewed. Only household incomes are determinant in explaining the probability of psychological abuse. With regards to the partners, their genders, nationalities, and levels of studies are important for determining the probability of physical, sexual, or psychological abuses. This work's empirical nature does not allow us to draw general conclusions and the results obtained are valid exclusively for the analyzed population (Spain, in our case). However, the available digital media have favored globalization in many topics, e.g., behavior patterns; for that reason, we would dare to say, with the caution that the matter deserves, that probably the conclusions reached could serve to understand violence sexual, at least, in countries with a level of development similar to that of Spain.

Two issues would deserve to be investigated: (a) a simultaneous study of the effect of covariates on the three types of abuse to see whether there are marginally significant differences between the results obtained in this study and those ones that could be derived from a multivariate study. (b) Some of the covariates used (such as jealous) also show asymmetry and their effect on the skewed response variable could also be studied.

\section{Declaration of Conflicting Interests}

The author(s) declared no potential conflicts of interest with respect to the research, authorship, and/or publication of this article.

\section{Funding}

The author(s) disclosed receipt of the following financial support for the research, authorship, and/or publication of this article: J.M.P.S. and E.G.D. were supported by the Ministerio de Economía, Industria y ompetitividad, Agencia Estatal de Investigación (Project ECO2017-85577-P). 


\section{ORCID iD}

José María Pérez-Sánchez (iD https://orcid.org/0000-0002-7491-4345

\section{References}

Aizer, A. (2010). The gender wage gap and domestic violence. American Economic Review, 100(4), 1847-1859.

Akaike, H. (1974). A new look at the statistical model identification. IEEE Transactions on Automatic Control, 19(6), 716-723.

Albert, J., \& Chib, S. (1995). Bayesian residual analysis for binary response regression models. Biometrika, 82(4), 747-769.

Allen, C. T., Carlson, J., Casey, E. A., Tolman, R., \& Leek, C. (2018). Examining men's perceptions of GBV prevention programming content. Violence Against Women, 25(5), 614-632.

Alonso-Borrego, C., \& Carrasco, R. (2017). Employment and the risk of domestic violence: Does the breadwinner's gender matter? Applied Economics, 49(50), 5074-5091.

Bernardo, J. (2004). Reference posterior distributions for Bayesian inference (with discussion). Journal of the Royal Statistical Society, Series B, 41(2), 113-147.

Bhattacharyya, M., Bedi, A., \& Chhachhi, A. (2011). Marital violence and women's employment and property status: Evidence from North Indian villages. World Development, 39(9), 1676-1689.

Brooks, C. (2009). RATS handbook to accompany introductory econometrics for finance. Cambridge University Press.

Capaldi, D. M., Knoble, N. B., Short, J. W., \& Kim, H. K. (2008). A systematic review of risk factors for intimate partner violence. Partner Abuse, 3(2), 231-280.

Chen, M., Dey, D., \& Shao, Q. (1999). A new skewed link model for dichotomous quantal response data. Journal of the American Statistical Association, 94(448), 1172-1186.

Famoye, F., \& Singh, K. P. (2006). Zero-inflated generalized Poisson regression model with an application to domestic violence data. Journal of Data Science, 4, 117-130.

Farmer, A., \& Tiefenthaler, J. (1997). An economic analysis of domestic violence. Review of Social Economy, 55(3), 337-358.

Gelman, A., Jakulin, A., Pittau, G., \& Su, Y.-S. (2008). A weakly informative default prior distribution for logistic and other regression models. The Annals of Applied Statistics, 2(4), 1360-1383.

Goodey, J. (2017). Violence against women: Placing evidence from a European Union wide survey in a policy context. Journal of Interpersonal Violence, 32(12), 1760-1791.

Haining, R. P., \& Li, G. (2020). Modelling spatial and spatial-temporal data: A Bayesian approach. Chapman \& Hall/CRC. 
Hartigan, J. (2014). Invariant prior distributions. Annals of Mathematical Statistics, 35(2), 836-845.

Hoeting, J., Madigan, D., Raftery, A., \& Volinsky, C. (1990). Bayesian model averaging: A tutorial. Statistical Science, 14(4), 382-417.

Jeffreys, H. (1961). Theory of probability (3rd ed.). Oxford University Press.

Jiang, X., Dey, D. K., Prunier, R., Wilson, A., \& Holsinger, K. E. (2013). A new class of flexible link functions with applications to species co-occurrence in cape floristic region. The Annals of Applied Statistics, 7(4), 2180-2204.

Kass, R., \& Wasserman, L. (1996). The selection of prior distributions by formal rules. Journal of the American Statistical Association, 91(435), 1343-1370.

Kim, S., Chen, M., \& Dey, D. (2008). Flexible generalized $t$-link models for binary response data. Biometrika, 95(1), 93-106.

Koop, K. (2003). Bayesian econometrics. John Wiley \& Sons.

Lemonte, A., \& Baz'an, J. (2018). New links for binary regression: An application to coca cultivation in Peru. Test, 27(3), 597-617.

Spiegelhalter, D., Best, N., Carlin, B., \& Van der Linde, A. (2002). Bayesian measures of model complexity and fit (with discussion). Journal of the Royal Statistical Society, Series B, 64(4), 583-639.

Spiegelhalter, D., Best, N., Carlin, B., der Linde, V A. (2014). The deviance information criterion: 12 years on. Journal of the Royal Statistical Society, Series B (Statistical Methodology), 76(3), 485-493.

Spiegelhalter, D., \& Smith, A. (2002). Bayes factors for linear and log-linear models with vague prior information. Journal of the Royal Statistical Society, Series B, 44(3), 377-389.

Wang, X., \& Dey, D. (2010). Generalized extreme value regression for binary response data: An application to B2B electronic payments system adoption. Annals of Applied Statistics, 4(4), 2000-2023.

Yodanis, C. (2004). Gender inequality, violence against women, and fear a crossnational test of the feminist theory of violence against women. Journal of Interpersonal Violence, 20(1), 7-11.

Zellner, A. (1996). An introduction to Bayesian inference in econometrics. Wiley.

\section{Author Biographies}

José María Pérez-Sánchez, $\mathrm{PhD}$, is an associate professor in the Faculty of Economics at University of Las Palmas de G.C., Spain. His research focuses on violence against women including sexual, psychological, and physical. He is interested in using Bayesian econometrics for incorporating the experts' opinion into the decision-making process.

Nancy Dávila-Cárdenes, $\mathrm{PhD}$, is an associate professor in the Faculty of Economics at University of Las Palmas de G.C., Spain. Her research focuses on violence against women including sexual, psychological, and physical. She is interested in quantitative tools for exploring and analyzing databases. 
Emilio Gómez-Déniz, PhD, is a professor in the Faculty of Economics at University of Las Palmas de G.C., Spain. His studies analyze Bayesian methodologies applied to VAW, actuarial science, sports, etc. Furthermore, his research also deepens the generation of new probability distributions to model the beliefs of an expert. 